\title{
Comparação da Qualidade de Vida Relacionada à Saúde entre Mulheres na Pós-menopausa Praticantes de Atividade Física com e sem Osteoporose
}

\section{Comparison of the Health-related Quality of Life between Physically Active Postmenopausal Women with or without Osteoporosis}

\author{
Marcelo Tavella Navega ${ }^{(1)}$, Jorge Oishi ${ }^{(2)}$
}

\section{RESUMO}

Objetivo: comparar a qualidade de vida relacionada à saúde de mulheres na pós-menopausa praticantes de atividade física com e sem o diagnóstico de osteoporose. Métodos: trata-se de estudo descritivo transversal, realizado por meio de entrevistas, em que o pesquisador aplicou uma ficha de avaliação e o questionário SF-36. Todas as voluntárias mulheres participaram do estudo por meio de um contato prévio por telefone. As características e objetivos da entrevista foram explicadas, além de confirmar o estágio pós-menopausa da voluntária. Formaram-se dois grupos, com 21 voluntárias cada um: grupo 1 , constituído por mulheres sem osteoporose $(64,38 \pm 4,24$ anos $)$, e grupo 2, constituído por mulheres com osteoporose $(67,81 \pm$ 4,19 anos). Cada voluntária, inicialmente, submetia-se a uma ficha de avaliação para obtenção dos dados pessoais, história clínica, comorbidades e cuidados com a saúde. Em seguida, aplicava-se o questionário SF-36. Para avaliação das diferenças entre os grupos, aplicou-se o teste não-paramétrico de Wilcoxon. Resultados: houve diferença significativa $(\mathrm{p}<0,05)$ entre os grupos em relação aos componentes Aspectos Físicos e Estado Geral da Saúde do SF-36, com desempenho melhor para o grupo 1. Conclusão: mulheres na pós-menopausa com diagnóstico de osteoporose, mas que não tiveram fraturas, podem ter qualidade de vida semelhante à de mulheres na pós-menopausa sem osteoporose.

Palavras-chave: qualidade de vida, pós-menopausa, SF-36, osteoporose.

\begin{abstract}
Objective: to investigate the quality of life among physically active post-menopausal women with and without a diagnosis of osteoporosis. Methods: a cross-sectional descriptive study was carried out through interview. All the participating women volunteered to take part of this study through previous contact by telephone. The characteristics and objectives of the interview were explained in addition to confirmation of their postmenopausal status. There were two groups of 21 volunteers each: group 1 were non-osteoporotic women $(64.38 \pm 4.24$ years-old) and group 2 were osteoporotic women (67.81 \pm 4.19 years-old). Each volunteer was asked to fulfill a preliminary form in order to register personal information, clinical history, co-morbidities and health care. Following, the SF-36 questionnaire was applied. The Wilcoxon rank-sum test was used to assess differences between the two groups. Results: there was a significant difference $(p<0.05)$ between both groups only with regard to the "Role Physical" and "General Health" components of the SF-36 form showing a better performance to group 1. Conclusion: post-menopausal women with a diagnosis of osteoporosis that did not sustain a fracture may present a similar quality of life, as compared to non-osteoporotic postmenopausal women.
\end{abstract}

Keywords: quality of life, post-menopausal women, SF-36, osteoporosis.

Recebido em 27/1 1/06. Aprovado, após revisão, em 19/06/07. Declaramos a inexistência de conflitos de interesse.

Trabalho desenvolvido no Departamento de Fisioterapia da Universidade Federal de São Carlos (UFSCar)

1. Doutor em Fisioterapia pela Universidade Federal de São Carlos (UFSCar) e professor-assistente da Universidade Estadual Paulista (Unesp), campus Marília

2. Doutor em Saúde Pública pela Universidade de São Paulo (USP) e professor adjunto do Departamento de Estatística e Programa de Pós-graduação em Fisioterapia da Universidade Federal de São Carlos (UFSCar)

Endereço para correspondência: Marcelo Tavella Navega, Rua Augusto Genta, 961, CEP 17519-340, Marília, SP, Brasil, e-mail: navegamt@marilia.unesp.br 


\section{INTRODUÇÃO}

O aumento acentuado do número de idosos nas últimas décadas e o fato de grande número deles permanecer em atividade e produzindo fizeram que o interesse pelo estudo do envelhecimento fosse se desenvolvendo progressivamente. Tanto os problemas de saúde dos idosos quanto os vários aspectos relativos à qualidade de vida dessa população tornaram-se objetos de preocupação e de estudos ${ }^{(1)}$.

Segundo o Instituto Brasileiro de Geografia Estatística (IBGE), a projeção da população no Brasil mostra a tendência de crescimento do número de idosos, que deve alcançar 25 milhões de pessoas em 2020, a maioria composta por mulheres (aproximadamente 15 milhões) ${ }^{(2)}$.

O conjunto de alterações estruturais e funcionais do organismo que se acumulam de forma progressiva, especialmente em função da idade, prejudica o desempenho de habilidades motoras, dificultando a adaptação do indivíduo ao meio ambiente e desencadeando modificações de ordem psicológica e social ${ }^{(3)}$. Com o avanço da idade, ocorre um declínio não linear da capacidade funcional dos diversos sistemas. O sistema ósseo sofre grande influência das alterações hormonais impostas pela menopausa, resultando em um processo de reabsorção óssea maior que o processo de formação, levando à diminuição fisiológica da massa óssea. Quando esse processo torna-se mais intenso, pode resultar no aparecimento de osteoporose, caracterizada por baixa massa óssea e deterioração da microarquitetura, aumentando a fragilidade óssea ${ }^{(4)}$.

A osteoporose tem sido recentemente reconhecida como um dos maiores problemas de saúde pública ${ }^{(5)}$. Atingindo cerca de um terço das mulheres na pós-menopausa, tornouse uma das doenças osteometabólicas mais comuns, sendo responsável por alto índice de morbidade e mortalidade entre os idosos, com enormes repercussões sociais e econômicas, provocando grande impacto na qualidade de vida e grau de independência nos indivíduos acometidos ${ }^{(6)}$.

A osteoporose é um problema clínico e social, pois pode dificultar o desenvolvimento das atividades cotidianas, influenciando o bem-estar e a qualidade de vida relacionada à saúde $(\mathrm{QVRS})^{(7)}$. Outro fator que pode influenciar a QVRS é a presença de comorbidades, fato que tende a aumentar com o passar dos anos de vida ${ }^{(8)}$. A prática regular de atividade física possibilita a manutenção ou até mesmo a melhora do estado de saúde física e psíquica de indivíduos de qualquer idade, inclusive de pacientes com osteoporose ${ }^{(1)}$. A medida da QVRS em pacientes com osteoporose é váli- da no intuito de determinar as mudanças necessárias para obtenção do bem-estar do paciente ${ }^{(9)}$.

O objetivo deste trabalho foi comparar a qualidade de vida de mulheres na pós-menopausa, praticantes de atividade física, com e sem osteoporose, contribuindo assim para um melhor entendimento e, conseqüentemente, favorecendo futuros planejamentos de condutas eficazes para melhora da qualidade de vida de mulheres com osteoporose.

\section{MÉTODO}

Trata-se de estudo descritivo transversal realizado por meio de entrevistas agendadas com voluntárias que fizeram o exame de densitometria óssea, nos anos de 2004 e 2005, em um instituto de diagnóstico por imagem na cidade de São Carlos, estado de São Paulo (Brasil), e que participavam regularmente de algum grupo de atividade física voltado à população idosa.

\section{SUJEITOS}

\section{RECRUTAMENTO}

Realizou-se o recrutamento com a utilização dos dados cadastrais de um programa de atenção à população idosa da cidade de São Carlos (SP). Efetuou-se o contato com as voluntárias previamente por telefone, no qual o pesquisador informava a característica e os objetivos da entrevista. Após o contato prévio com aproximadamente 200 mulheres, formaram-se dois grupos, com 21 voluntárias cada um, sendo o grupo 1 constituído por mulheres sem osteoporose $(64,38 \pm 4,24$ anos) e o grupo 2 constituído por mulheres com osteoporose $(67,81 \pm 4,19$ anos $)$. As demais mulheres não participaram do estudo, pois não aceitaram ou não compareceram à entrevista agendada.

Para o diagnóstico de osteoporose, utilizou-se o valor de densidade mineral óssea ( $\mathrm{T}$ ) inferior a - 2,5 desvios-padrão (DP) na coluna vertebral e/ou fêmur.

O trabalho foi aprovado pelo Comitê de Ética em Pesquisa (CEP) da Universidade Federal de São Carlos (UFSCar) (protocolo no 079/04). As candidatas foram informadas sobre as características do estudo e aceitaram participar voluntariamente, conforme determina a resolução 196/96 do CNS.

\section{CRITÉRIOS DE INCLUSÃo E EXCLUSÃo}

Incluíram-se todas que confirmaram o período pósmenopausa, com pelo menos 12 meses de intervalo da última menstruação ${ }^{(10)}$, praticantes de atividade física havia pelo menos 1 ano e que não tinham diagnóstico de fratura 
por fragilidade óssea e que aceitaram participar da pesquisa. Seriam excluídas as voluntárias que apresentassem algum comprometimento que dificultasse responder às avaliações propostas. Entretanto, não foi necessário utilizar esse critério de exclusão.

\section{PROCEDIMENTO}

Realizaram-se as entrevistas na sala de avaliação do Ambulatório de Fisioterapia da Universidade Federal de São Carlos, de forma individual, com duração média de 70 minutos. Cada voluntária, inicialmente, submetia-se a uma ficha de avaliação para obtenção dos dados pessoais, aspecto socioeconômico, história clínica, comorbidades, estilos de vida e cuidados com a saúde. Em seguida, aplicava-se o questionário Medical Outcomes study 36 - Item ShortForm Health Survey (SF-36).

O questionário genérico SF-36 é um instrumento de medida da qualidade de vida relacionada à saúde $(\mathrm{QVRS})^{(11)}$. Esse questionário é mundialmente utilizado e possui validação em mais de 15 países $^{(7)}$. No Brasil, Ciconelli et al. ${ }^{(12)}$ realizaram a versão do SF-36 para a língua portuguesa.

O SF-36 é um questionário multidimensional formado por 36 itens, englobados em 8 escalas (componentes): Capacidade Funcional, Aspectos Físicos, Dor, Estado Geral de Saúde, Vitalidade, Aspectos Sociais, Aspectos Emocionais e Saúde Mental. Cada um desses componentes possui um escore, cuja pontuação varia de 0 a 100 , sendo zero o pior estado de saúde e 100 o melhor estado de saúde.

$\mathrm{Na}$ avaliação de comorbidades, utilizou-se a Cumulative Illness Rating Scale (CIRS), que investiga a presença de 14 "conjuntos" de doenças (cardíaca, vascular, hematológica, respiratória, oftalmológica, gastrointestinal alta, gastrointestinal baixa, hepática e pancreática, renal, geniturinária, musculoesquelética e tegumentar, neurológica, endócrina e metabólica e de mama, e psiquiátrica), considerando as situações de ausência, leve, moderada, severa ou extremamente severa para cada "conjunto" de doenças, recebendo a pontuação $0,1,2,3$ ou 4 , respectivamente ${ }^{(13)}$.

\section{ANÁLISE dOS DADOS}

Os dados foram expressos em média e desvio-padrão. Para avaliação das diferenças entre os grupos, aplicou-se o teste não-paramétrico de Wilcoxon. Calculou-se a consistência interna das medidas obtidas por SF-36 e CIRS pelo coeficiente de correlação de Spearman. O nível de significância utilizado para as conclusões das análises estatísticas foi de $5 \%(\mathrm{p} \leq 0,05)$.

\section{RESULTADOS}

Na Tabela 1, ilustra-se a caracterização dos grupos em relação aos dados antropométricos, período pós-menopausa, dados sobre comorbidades e resultados da última densitometria óssea.

TABELA 1

CARACTERÍSTICAS CLÍNICAS E DEMOGRÁFICAS DAS MULHERES COM E SEM OSTEOPOROSE

\begin{tabular}{lccc}
\hline Características & $\begin{array}{c}\text { Grupo 1 } \\
(\mathbf{n}=\mathbf{2 1})\end{array}$ & $\begin{array}{c}\text { Grupo 2 } \\
(\mathbf{n}=\mathbf{2 1})\end{array}$ & Valor de $\mathbf{p}$ \\
\hline Idade (anos) & $64,38 \pm 4,24$ & $67,81 \pm 4,19$ & $0,0119^{*}$ \\
\hline $\begin{array}{l}\text { Período } \\
\text { pós-menopausa (anos) }\end{array}$ & $18,95 \pm 10,96$ & $22,10 \pm 6,32$ & 0,0646 \\
\hline Estatura (m) & $1,60 \pm 0,05$ & $1,53 \pm 0,07$ & $0,0037^{*}$ \\
\hline $\begin{array}{l}\text { Peso (kg) } \\
\text { Indice de massa } \\
\left.\text { corporal (kg/m }{ }^{2}\right)\end{array}$ & $66,01 \pm 8,76$ & $57,60 \pm 9,19$ & $0,0037^{*}$ \\
\hline $\begin{array}{l}\text { Densidade mineral da } \\
\text { coluna lombar (g/cm }{ }^{2} \text { ) }\end{array}$ & $1,204 \pm 0,116$ & $0,857 \pm 0,119$ & $0,0000^{*}$ \\
\hline $\begin{array}{l}\text { T-escore (coluna lombar) } \\
\text { Comorbidades } \\
\text { (valores da CIRS) }\end{array}$ & $-1,14 \pm 0,70$ & $-3,15 \pm 0,83$ & $0,0000^{*}$ \\
\hline \begin{tabular}{l} 
Número de comorbidades \\
\hline
\end{tabular} & $2,8,52 \pm 3,52$ & $11,62 \pm 6,38$ & 0,0605 \\
\hline
\end{tabular}

Dados estão expressos como média \pm desvio-padrão.

${ }^{*} p<0,05$ (significância estatística).

CIRS $=$ Cumulative Illness Rating Scale.

Grupo 1 = sem osteoporose; grupo 2 = com osteoporose.

A quantidade e a intensidade de comorbidades não apresentaram diferenças significativas entre os grupos. Para ambos os grupos, as comorbidades mais apontadas foram: hipertensão arterial sistêmica, dislipidemia, diabetes do tipo II, gastrite e osteoartrose. Desta forma, ao comparar a qualidade de vida relacionada à saúde, entre os grupos, a única diferença preponderante era a presença ou ausência de osteoporose.

Todas as voluntárias praticavam atividade física regular, de três a cinco vezes por semana, com duração média de 60 minutos cada sessão. Esse nível de atividade está além do mínimo de atividade física recomendada pelo American College of Sports Medicine ${ }^{(14)}$. Atividade aeróbia (caminhada) e alongamentos gerais foram realizados por todas as voluntárias (Tabela 2 ).

A Figura 1 apresenta os resultados referentes ao questionário SF-36 que avalia a qualidade de vida relacionada à saúde. Pode-se observar que apenas os componentes Aspectos Físicos e Estado Geral da Saúde apresentaram diferenças significativas entre os grupos. 
TABELA 2

CaracterizaÇÃo DA AMOSTRA QuANTO À REALIZAČ̃̃ DE ATIVIDADE FÍSICA

\begin{tabular}{lcc}
\hline Atividade física & $\begin{array}{c}\text { Grupo 1 } \\
(\mathbf{n = 2 1 )}\end{array}$ & $\begin{array}{c}\text { Grupo 2 } \\
(\mathbf{n}=\mathbf{2 1})\end{array}$ \\
\hline Caminhada & $100 \%$ & $100 \%$ \\
\hline Alongamentos gerais & $100 \%$ & $100 \%$ \\
\hline Hidroginástica & $28,57 \%$ & $33,33 \%$ \\
\hline Exercício resistido & $14,29 \%$ & $9,52 \%$ \\
\hline Natação & $4,76 \%$ & $4,76 \%$ \\
\hline
\end{tabular}

Valores expressos em porcentagem.

Consideraram-se as atividades realizadas na frequiência mínima de três vezes por semana.

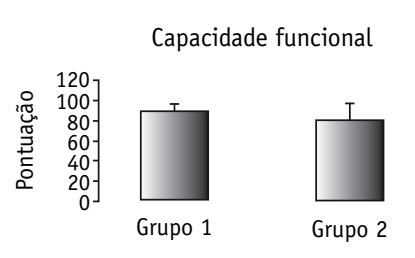

Dor
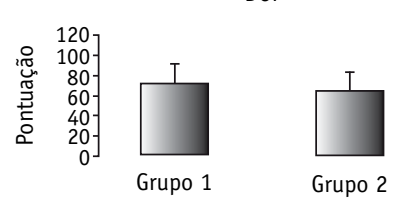

Vitalidade

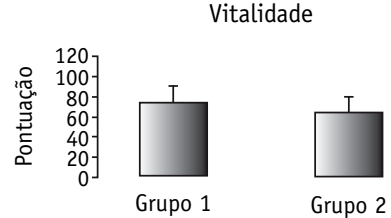

Aspectos emocionais
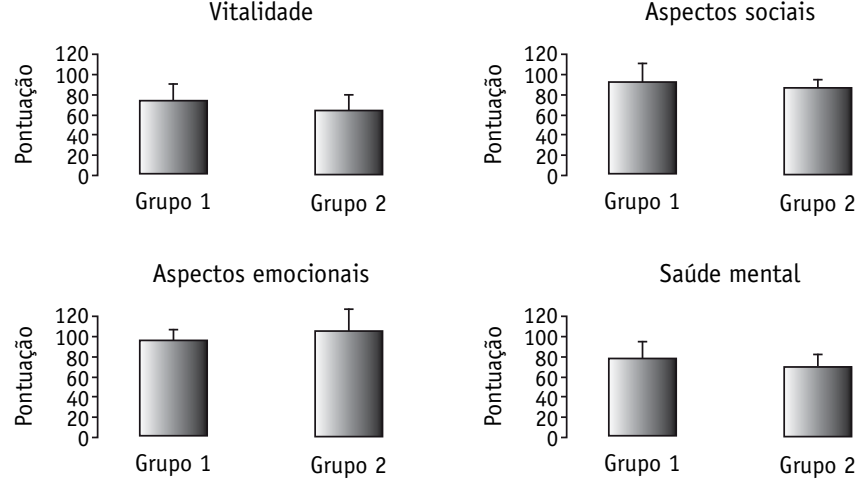

Grupo 1 = sem osteoporose $(n=21) ;$ grupo $2=$ com osteoporose $(n=21)$.

* Diferença estatística $(p<0,05)$

Figura 1 - Pontuação do SF-36 das mulheres com e sem osteoporose.

Na Tabela 3, ilustra-se o resultado da correlação entre as medidas obtidas por SF-36 e CIRS para ambos os grupos. Pode-se observar que, de forma geral, a pontuação do questionário de qualidade de vida relacionada à saúde não apresentou correlação com a pontuação referente a comorbidades. Para o grupo de mulheres com osteoporose, os componentes Capacidade Funcional, Aspectos Físicos, Estado Geral de Saúde e Aspectos Sociais apresentaram correlações significativas. O grupo de mulheres sem osteoporose apresentou correlações significativas nos componentes Aspectos Físicos, Dor e Aspectos Sociais. Para ambos os grupos, as correlações foram fracas $(r<0,5)$, sem relevância clínica ${ }^{(15)}$.

TABELA 3

Coeficientes de Correlação de Spearman entre os DOMÍNIOS DO SF-36 COM A MEDIDA DE COMORBIDADE NAS MULHERES COM E SEM OSTEOPOROSE

\begin{tabular}{lcccc}
\hline & \multicolumn{2}{c}{ Grupo $\mathbf{1}(\mathbf{n}=\mathbf{2 1})$} & \multicolumn{2}{c}{ Grupo $2(\mathbf{n}=\mathbf{2 1})$} \\
Componentes & $\mathbf{r}$ & Valor de $\mathbf{p}$ & $\mathbf{r}$ & Valor de $\mathbf{p}$ \\
\hline Capacidade funcional & $-0,240$ & 0,063 & $-0,260$ & $0,01^{*}$ \\
\hline Aspectos físicos & $-0,300$ & $0,008^{*}$ & $-0,089$ & $0,011^{*}$ \\
\hline Dor & $-0,350$ & $0,000^{*}$ & 0,370 & 0,367 \\
\hline Estado geral de saúde & $-0,083$ & 0,088 & $-0,390$ & $0,003^{*}$ \\
\hline Vitalidade & 0,030 & 0,093 & 0,064 & 0,099 \\
\hline Aspectos sociais & $-0,351$ & $0,016^{*}$ & $-0,130$ & $0,035^{*}$ \\
\hline Aspectos emocionais & 0,139 & 0,112 & 0,152 & 0,410 \\
\hline Saúde mental & 0,297 & 0,642 & 0,148 & 0,232 \\
\hline
\end{tabular}

${ }^{*} p<0,05$.

\section{DISCUSSÃO}

As voluntárias que aceitaram participar apresentavam-se com uma condição de saúde satisfatória, independentemente de terem ou não osteoporose. Pode-se sugerir que boa condição de saúde reflita maior tendência à participação de pesquisas deste gênero, ou seja, é mais provável que uma pessoa que esteja se sentindo bem aceite participar de uma atividade não corriqueira do que uma pessoa que acredita que a sua condição de saúde esteja aquém da média da população. Além disso, todas as voluntárias realizavam atividades voltadas à terceira idade, o que resultou em uma amostra de mulheres ativas fisicamente, o que não é comumente encontrado na população idosa, que apresenta elevada porcentagem de sedentários ${ }^{(1)}$.

O grupo de pacientes com osteoporose era mais idoso do que o grupo de mulheres na pós-menopausa sem osteoporose, com uma diferença na média de idade de um pouco mais de 3 anos. Tal fato era esperado, pois a osteoporose tende a apresentar maior incidência em populações mais senis ${ }^{(16)}$. 
Em relação aos dados antropométricos, apesar das diferenças significativas entre os grupos quanto aos valores de estatura e massa corporal, o índice de massa corporal (IMC) apresentou-se sem diferença, o que indica que a distribuição de massa corporal não se diferenciou entre mulheres com e sem osteoporose. Em ambos os grupos, os valores médios do IMC estão na transição entre composição corporal normal e sobrepeso, o que pode favorecer perda de massa óssea menos acelerada após a menopausa ${ }^{(17)}$. A avaliação das comorbidades apresentou baixa correlação com a qualidade de vida relacionada à saúde. Dados similares foram obtidos por Fortin et al. ${ }^{(8)}$, que observaram valores baixos de correlação (de - 0,55 a - 0,18) entre os componentes do SF-36 e da CIRS. Pesquisas que usaram escalas designadas para prognosticar mortalidade concluíram que comorbidades apresentam baixa correlação com incapacidade física ${ }^{(18,19)}$.

Os componentes avaliados pelo SF-36 mais afetados pela presença de osteoporose são Estado Geral de Saúde, Capacidade Funcional, Dor e Aspectos Físicos ${ }^{(7)}$. O componente Estado Geral de Saúde considera a percepção do indivíduo quanto à sua saúde e o grau de concordância de quatro afirmações a respeito da perspectiva da sua saúde e a comparação em relação a outras pessoas. As mulheres com osteoporose tendem a ter pior percepção da sua saúde do que as que não possuem grande perda de massa óssea, pois somente o diagnóstico de osteoporose já promove um impacto negativo na percepção de saúde, provocada pelo receio de incapacidade e fraturas ${ }^{(20)}$.

O componente Aspectos Físicos é formado por quatro questões que identificam o impacto da saúde física no trabalho ou em alguma atividade diária regular durante as últimas 4 semanas. As médias obtidas tanto pelas mulheres sem osteoporose $(94,05)$ quanto pelas com osteoporose $(77,38)$ são consideradas altas quando comparadas com os valores obtidos por Aranha et al. ${ }^{(7)}$, que avaliaram a QVRS de espanholas com osteoporose e que obtiveram a média de 59,9 nesse componente. Pode-se explicar a diferença significativa nesse componente entre as mulheres com e sem osteoporose pelos prejuízos físicos decorrentes da baixa densidade mineral óssea, que impõe maior risco de ocorrência de eventos prejudiciais à saúde e pode desencadear restrição na realização de atividades ${ }^{(21)}$.

A ausência de diferenças significativas nos componentes "Dor" e "Capacidade Funcional" pode ser explicada pelo fato de a amostra ter sido formada por mulheres praticantes de atividade física regular, o que é comprovadamente benéfico tanto para mulheres com osteoporose ${ }^{(20,22,23)}$ quanto para a população idosa em geral ${ }^{(1,24,25)}$. Esse dado reforça a necessidade de ampliação de programas de atenção aos idosos que ofereçam atividades físicas específicas para essa população, pois pode ser uma boa estratégia para manter idosos ativos fisicamente, minimizando a perda funcional desencadeada pelo processo de envelhecimento e acentuada pelo sedentarismo.

A realização de atividade física pode, entre outros fatores, melhorar a capacidade funcional ${ }^{(26)}$, diminuir a dor ${ }^{(27,28)}$, reduzir o uso de analgésicos ${ }^{(28)}$ e melhorar a qualidade de vida de indivíduos com osteoporose ${ }^{(20,27)}$.

No estudo realizado por Acree et al. ${ }^{(29)}$, que investigaram se a atividade física associa-se com qualidade de vida relacionada à saúde de idosos saudáveis, observou-se que idosos que participavam de atividade física regular de intensidade pelo menos moderada por mais de 1 hora semanal obtiveram valores mas altos nos oito domínios do SF-36 do que os idosos que realizavam menos atividade física.

Binder et al. ${ }^{(26)}$ obtiveram melhora da qualidade de vida, avaliada por meio do SF-36, do grupo que realizou um treinamento constituído de exercícios de flexibilidade, equilíbrio, coordenação, resistência e atividade aeróbia.

Chow e Harrison ${ }^{(30)}$, após submeterem indivíduos osteoporóticos a um programa de atividade física, constataram diminuição da dor e aumento da mobilidade e da capacidade funcional. Resultados similares foram verificados por Malmros et al. ${ }^{(28)}$, que observaram diminuição significativa do nível de dor e do uso de analgésico em mulheres osteoporóticas com dor crônica na coluna lombar, as quais se submeteram a um programa de atividade física supervisionado por fisioterapeutas. Dessa forma, o fato de as voluntárias deste estudo serem praticantes de atividade física regularmente pode ter colaborado para que não houvesse diferença no componente "Dor" entre as mulheres com e sem osteoporose. A diminuição da capacidade funcional e maiores níveis de dor relacionam-se mais diretamente à inatividade desencadeada pelo impacto psicológico ocasionado pelo diagnóstico de osteoporose do que a uma conseqüência física resultante da baixa densidade mineral óssea. Além disso, por o número e a intensidade das comorbidades serem semelhantes entre os grupos, e algumas delas possuírem características que podem influenciar a dor e a capacidade funcional dos pacientes, é possível que as comorbidades associadas com a prática regular de atividade física tenham sido mais preponderantes do que a presença ou não de osteoporose.

A osteoporose tem como principal conseqüência a fratura, que freqüentemente impõe severas limitações emocionais e sociais, comprometendo a qualidade de vida, em 
virtude da instalação de um quadro de ansiedade, depressão e mudança de humor ${ }^{(31)}$, além de favorecer os pacientes a adotar um estilo de vida sedentário e mais isolado socialmente, permanecendo mais tempo dentro de casa não só pela dor, mas também pelo medo de novas fraturas ${ }^{(22)}$. Desta forma, o que mais contribui para uma pior qualidade de vida em indivíduos acometidos por osteoporose é a presença de fraturas relacionadas à osteoporose e não somente a osteoporose. Tal fato reforça a necessidade de medidas educativas e preventivas; primeiro, na tentativa de diminuir o número

\section{REFERÊNCIAS}

1. Rebelatto JR, Calvo JI, Arejuela JR, Portillo JC: Influência de um programa de atividade física de longa duração sobre a força muscular manual e a flexibilidade corporal de mulheres idosas. Rev Bras Fisioter 10 (1): 127-32, 2006.

2. Parahyba MI, Veras R, Melzer D: Disability among elderly women in Brazil. Rev Saúde Pública 39 (3): 383-91, 2005.

3. Meirelles MEA: Atividade física na $3^{a}$ idade. $3^{a}$ ed. Rio de Janeiro: Sprint, 2000.

4. Ritson F, Scott S: Physiotherapy for osteoporosis: a pilot study comparing practice and Knowledge in Scotland and Sweden. Physiotherapy 82 (7): 1390-4, 1996.

5. Engermann M, Schneider E, Evans CH, Baltzer AW: The potential of gene therapy for fracture healing in osteoporosis. Osteoporos Int 15 (1): 82-7, 2005.

6. Forsbach G, Santos A: Densidad óssea y osteporosis: una opinion. Ginecol Obstet 62: 201-3, 1994.

7. Aranha LLM, Mirón Canelo JA, Alonso Sardón M, Del Pino Montes J, Sáenz González MC: Qualidade de vida relacionada à saúde em espanholas com osteoporose. Rev Saúde Pública 40 (2): 298-303, 2006.

8. Fortin M, Hudon C, Dubois MF, Almirall J, Lapointe L, Soubhi H: Comparative assessment of three different indices of multimorbidity for studies oh health-relate quality of life. Healh Qual Life Outcomes 3 (74): 1-7, 2005.

9. Cvijetic S, Mestrovic T, Crkevenac A, Davila S, Korsic M: Quality of live in osteoporotic patients with hip fracture and without fracture. Arh Hig Rada Toksikol 53 (4): 257-62, 2002.

10. Freitas EV, Pimenta L: Climatério In: Freitas EV, et al. Tratado de geriatria e gerontologia. Rio de Janeiro: Guanabara-Koogan, 2002.

11. Ware JE Jr, Sherbourne CD: The MOS 36-item short-form survey (SF-36). Conceptual framework and item selection. Med Care 30: 473-83, 1992.

12. Ciconelli RM, Ferraz MB, Santos W, Meinão I, Quaresma MR: Tradução para a língua portuguesa e validação do questionário genérico de avaliação de qualidade de vida SF-36 (Brasil SF-36). Rev Bras Reumatol 39 (3): 143-50, 1999.

13. Fortin M, Hudon C, Dubois MF, Almirall J, Lapointe L, Soubhi H: Comparative assessment of three different indices of multimorbidity for studies oh health-relate quality of life. Healh Qual Life Outcomes 3 (74): 1-7, 2005. de pacientes com osteoporose, e segundo, para intervir o mais precocemente possível em favor dos pacientes com osteoporose, para que estes permaneçam ativos fisicamente e mantenham-se sem sofrer fraturas.

Nossos dados permitem concluir que mulheres na pós-menopausa acometidas por osteoporose, mas que não tenham diagnóstico de fraturas ocorridas por baixo impacto e que pratiquem regularmente atividade física, têm qualidade de vida semelhante à de mulheres na pós-menopausa sem osteoporose.

14. Pate RR, Pratt M, Blair SN, et al.: Physical activity and public health: a recommendation from the Centers for Disease Control and Prevention and the American College of Sports Medicine. JAMA 273: 402-7, 1995.

15. Youm T, Koval KJ, Zuckerman JD: The economic impact of geriatric hip fractures. Am J Orthop 28 (7): 423-8, 1999.

16. Melton LJ, Riggs BL: Epidemiology and cost of osteoporotic fractures. In: International Conference on Osteoporosis. Milano, 1996.

17. Oishi J, Rennó ACM, Navega MT, Driusso P: Obesidade e osteoporose. In: Damaso A. Obesidade. Rio de Janeiro: Medsi, 2003.

18. Extermann M, Overcash J, Lyman G, Parr J, Balducci L: Comorbidity and functional status are independent in older cancer patients. J Clin Epidemiol 53: 1258-67, 2000.

19. Repetto L, Fratino L, Audisio RA, et al.: Comprehensive geriatric assessment the information to Easterm Cooperative Oncology Group performance status in elderly cancer patients: an Italian Group for Geriatric Oncology Study. J Clin Oncol 20 (2): 494-502, 2002.

20. Aveiro MC, Navega MT, Granito RN, Rennó ACM, Oishi J: Efeitos de um programa de atividade física no equilíbrio e na força muscular do quadríceps em mulheres osteoporóticas visando a uma melhoria na qualidade de vida. Rev Bras de Ciência e Movimento 12 (3): 33-8, 2004.

21. Driusso P. Efeitos de um programa de atividade física na qualidade de vida de mulheres com osteoporose. Universidade Federal de São Carlos. Dissertação (Mestrado em Fisioterapia), 2000.

22. Rennó ACM, Granito RN, Driusso P, Costa D, Oishi J. Efeccts of an exercise program on respiratory function, posture and on quality of life in osteoporotic women: a pilot study. Physiotherapy 91: 113-8, 2005.

23. Navega MT, Aveiro MC, Oishi J. Alongamento, caminhada e fortalecimento dos músculos da coxa: um programa de atividade física para mulheres com osteoporose. Rev Bras de Fisioter 7 (3): 261-7, 2003.

24. Hauer K, Rost B, Rütschle K, et al.: Exercise training for rehabilitation and secondary prevention of falls in geriatric patients with a history of injurious falls. JAGS 49:10-20, 2001.

25. Kerschan K, Alacamlioglu Y, Kollmitzer J, et al.: Functional impact of unvarying exercise program in women after menopause. Am J Phys Med Rehabil 77: 326-32, 1998. 
26. Binder EF, Schechtman KB, Ehsani AA, et al.: Effects of exercise training on frailty in community-dwelling older adults: results of a randomized, controlled trial. JAGS 50 (12): 1921-8, 2002.

27. Granito RN, Rennó ACM, Aveiro MC, Navega MT, Driusso P, Oishi J: Efeitos de um programa de atividade física na postura hipercifótica torácica, na dorsalgia e na qualidade de vida de mulheres com osteoporose. Rev Bras Fisioter 8 (3): 231-7, 2004.

28. Malmros B, Mortensen L, Jensen MB, Charles P: Positive effects of physiotherapy on chronic pain and performance in osteoporosis. Osteoporosis Int 8: 215-21, 1998.
29. Acree LS, Longfors J, Fjeldstad AS, et al.: Physical activity is related to quality of life in older adults. Health and Quality of Life Outcomes 4 (37): 1-6, 2006.

30. Chow KR, Harrison EJ: Relationship of khyphosis to physical fitness and bone mass on post-menopause women. American Journal of Physical Medicine 66 (5): 219-27, 1989.

31. Galsworthy TD, Wilson PL: Osteoporosis: it steals more than bone. Am J Nurs 96: 27-33, 1996. 